Jean Bénard

Olivier Hartmann

Marie-José

Terrier-Lacombe

Chantal Bayle

Jean Lemerle

Guy Riou

\section{Gènes impliqués dans la dissémination métastatique et la réponse à la chimiothérapie du neuroblastome}

Le neuroblastome est un cancer de l'enfant qui, lorsqu'il est métastatique d'emblée, a un très mauvais pronostic. Il est associé à de nombreuses altérations génétiques qui jouent un rôle déterminant dans le potentiel de dissémination métastatique : délétion du bras court du chromosome 1, amplification de l'oncogène $\mathrm{N}-M Y C$, absence d'expression du gène $C D 44$. La mise au point de modèles expérimentaux de neuroblastome humain chez la souris athymique a permis de caractériser un ensemble d'anomalies génétiques spécifiquement associées au potentiel de dissémination: surexpression d'oncogènes, répression ou mutations de gènes suppresseurs de tumeurs, activation de gènes de résistance multiple aux médicaments (MDR1 et GST- $\pi$ ) et activation de gènes antiapoptotiques. Ces anomalies génétiques des neuroblastomes métastatiques expliquent leur résistance à une chimiothérapie intensive, et permettent de proposer une nosologie de ces formes de cancer qui pourraient déboucher sur de nouvelles approches thérapeutiques spécifiques en clinique oncologique.

\section{ADRESSES}

J. Bénard: assistant, laboratoire de pharmacologie moléculaire. O. Hartmann: directeur de l'unité de transplantation médullaire. Département de pédiatrie. M.-J. Terrier-Lacombe: assistante. Département d'anatomie pathologique. C. Bayle: directrice du laboratoire d'hématologie. Département de biologie clinique. J. Lemerle: chef de service de pédiatrie. G. Riou: directeur du laboratoire de pharmacologie moléculaire. Institut Gustave Roussy, rue Camille-

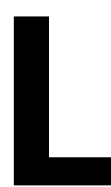

e neuroblastome, tumeur solide du jeune enfant, manifeste une grande hétérogénéité génétique. Les travaux récents de cytogénétique et de biologie moléculaire ont révélé dans les tumeurs primitives des altérations génétiques fréquentes qui permettent de définir des profils génétiques distincts. Cette caractérisation moléculaire, qui s'avère utile pour traiter spécifique- ment les formes localisées de la maladie, est actuellement en cours pour les formes métastatiques d'emblée chez l'enfant de plus d'un an. Nous développerons dans cette revue les principales avancées récemment rapportées dans ce domaine et qui concernent l'identification d'altérations génétiques spécifiquement associées aux neuroblastes malins métastatiques, la création de modèles expérimentaux 


\section{RÉFÉRENCES}

1. Hartmann O, Pein F, Caillaud JM, Bayle C, Couanet D, Gauthier F, Comoy E, Habrand JL. Neuroblastomes. Cancers de l'enfant (J. Lemerle). In: Hoerni B, Tubiana M, eds. Encyclopédie des Cancers 1989: 387427.

2. Hartmann O, Pinkerton C, Philip T, Zucker JM, Breatnach F. Very high-dose cis-platinum and etoposide in children with untreated advanced neuroblastoma. J Clin Oncol 1988; 6 : 44-50.

3. Fong C, Dracopoli N, White P, Merril P, Griffith R, Housman D, Brodeur G. Loss of heterozygosity for the short arm of chromosome 1 in human neuroblastoma with MYCN amplification. Proc Natl Acad Sci USA $1989 ; 86: 3753-7$.

4. Schwab M, Alitalo K, Klempnauer K, Varmus H, Bishop J, Gelbert F, Brodeur G, Goldstein M, Trent J. Amplified DNA with limited homology to myc cellular oncogene is shared by human neuroblastoma cell lines and a neuroblastoma tumour. Nature 1983 ; 305: 245-8.

5. Bourhis J, DeVathaire F, Wilson G, Hartmann O, Terrier-Lacombe MJ, BocconGibod L, McNally N, Lemerle J, Riou G, Bénard J. Combined analysis of DNA ploidy index and $\mathrm{N}-m y c$ genomic content in neuroblastoma. Cancer Res 1991 ; 51: 33-6.

6. Nakagawara A, Arima-Nakagawara M, Scavarda N, Azar C, Cantor A, Brodeur G. Association between high levels of expression of the TRK gene and favorable outcome in human neuroblastoma. $N$ Engl J Med 1993; 328: 847-54.

7. Versteeg R, Caron H, Cheng N, van der Drift P, Slater R, Wersterveld A, Voûte P, Delattre O, Laureys G, Van Roy N, Speleman F. 1p36 : every subband a suppressor ? Eur J Cancer 1995; 31A: 538-41.

8. Brodeur G. Molecular basis for heterogeneity in human neuroblastoma. Eur J Cancer 1995; 31A: 505-9.

9. Rubie H, Plantaz D, Michon J, Chastagner $\mathrm{P}$, Coze $\mathrm{C}$, Baranzelli MC, Avet-Loiseau $\mathrm{H}$, Gauthier F, Bénard J, Delattre O, Combaret V, Hartmann O. Localized neuroblastoma : N-myc is the main pronostic factor and post-operative treatment can be deleted in infants. SIOP XXVth Meeting Abstrats 1993, p110, N $^{\circ} 582$.

10. Bourhis J, Dominici C, McDowell H, Raschella G, Wilson G, Castello M, Plouvier E, Lemerle J, Riou G, Bénard J, Hartmann O. N-myc genomic content and DNA ploidy in stage-IVS neuroblastoma. J Clin Oncol 1991; $9: 1371-5$.

$\mathrm{m} / \mathrm{s} n^{\circ} 6-7$, vol. 12, juin-juillet 96 pour la détection de ces anomalies génétiques et, enfin, le rôle des gènes impliqués dans la réponse du neuroblastome à la chimiothérapie. On peut espérer que l'identification d'altérations génétiques spécifiquement associées au potentiel métastatique permettra d'améliorer la thérapeutique de ces formes très agressives de la maladie.

\section{Mise en évidence d'entités génétiques du neuroblastome}

Le neuroblastome est une tumeur embryonnaire du système neuroectodermique spécifique de l'enfant. Cette tumeur solide siège au niveau cervical, thoracique et surtout abdominal et se développe aux dépens du système sympathique (chaîne ganglionnaire et médullosurrénale). Les neuroblastomes sont, avec les tumeurs cérébrales, les tumeurs solides les plus fréquentes de l'enfant de moins de cinq ans (incidence de 1 pour 7000 enfants). La classification de la maladie, fondée sur des critères cliniques (taille de la tumeur primitive, présence ou non de métastases d'emblée) permet de différencier les formes localisées de stade I, II, III, des formes métastatiques d'emblée, de stade IV, formes qui définissent deux maladies cliniquement différentes.

La dissémination métastatique qui caractérise les neuroblastomes de stade IV est révélée au diagnostic. Les métastases se développent à distance de la tumeur primitive, essentiellement dans les tissus ostéo-médullaires, parfois dans les ganglions à distance et dans le foie. On ne peut établir de relation entre la taille de la tumeur primitive et la présence de métastases. Ce sont les bilans d'extension médullaire, cytologiques et histologiques, ainsi que le bilan scintigraphique osseux qui révèlent les métastases (figure 1). Une forme métastatique spécifique du nourrisson, le stade IV-S, caractérisée par une tumeur primitive de petit volume et une dissémination cutanée, hépatique, quelquefois médullaire mais jamais osseuse, peut, dans certains cas, régresser spontanément de façon spectaculaire.

Le pronostic clinique dépend à la fois de l'âge (plus favorable s'il est infé- rieur à un an, plus sévère s'il est supérieur à un an) et du stade clinique lors du diagnostic. Les neuroblastomes localisés sont traités essentiellement par chirurgie, ce qui permet une excellente survie. A l'inverse, le taux de survie est faible (30\% de survie à cinq ans) chez l'enfant de plus d'un an présentant un neuroblastome métastatique d'emblée (environ $50 \%$ des neuroblastomes au diagnostic), à l'exception du stade IV-S. Une chimiothérapie agressive associée ou non à une autogreffe de moelle osseuse ne modifie pas ce pronostic [1, 2] (figure 2).

Le développement des techniques de cytogénétique, cytofluorimétrie et biologie moléculaire a permis d'identifier, à partir des tumeurs primitives de patients, des altérations génétiques spécifiques du neuroblastome $\left(\mathrm{m} / \mathrm{s} n^{\circ} 4\right.$, vol. $\left.12, p .527\right)$. Ces altérations sont: (1) la perte d'hétérozygotie du bras court du chromosome 1 au niveau de la bande 1p36 [3]; (2) l'amplification de l'oncogène N-MYC, [4] ; (3) la perte d'hétérozygotie du bras long du chromosome 14; (4) le réarrangement du bras long du chromosome 17; (5) une hyperdiploïdie, avec un contenu en ADN des cellules tumorales augmenté d'environ $50 \%$ ou une diploïdie [5]; (6) une altération de l'expression ou de la fonction du récepteur du NGF (nerve growth factor) codé par l'oncogène TRKA. L'hyperdiploïdie et la surexpression de TRKA sont des facteurs de bon pronostic [6]. En revanche, la perte d'hétérozygotie en 1p36, qui correspond à l'inactivation d'un ou de plusieurs gènes suppresseurs de tumeurs [7], et l'amplification de l'oncogène $\mathrm{N}-M Y C$, sont deux facteurs génétiques souvent associés et de mauvais pronostic [7, 8]: ils sont retrouvés essentiellement dans les neuroblastomes métastatiques d'emblée des enfants de plus d'un an. La combinaison de quatre de ces facteurs a permis de définir trois types génétiques distincts de neuroblastome [8] (Tableau I). Le type I présente une hyperdiploïdie et une surexpression de TRKA, sans autres altérations. Le type II correspond à un contenu en ADN diploïde avec une expression de TRKA faible et, parfois, une délétion modérée en 1p36. Le type III est défini par un contenu en ADN diploïde, une perte 


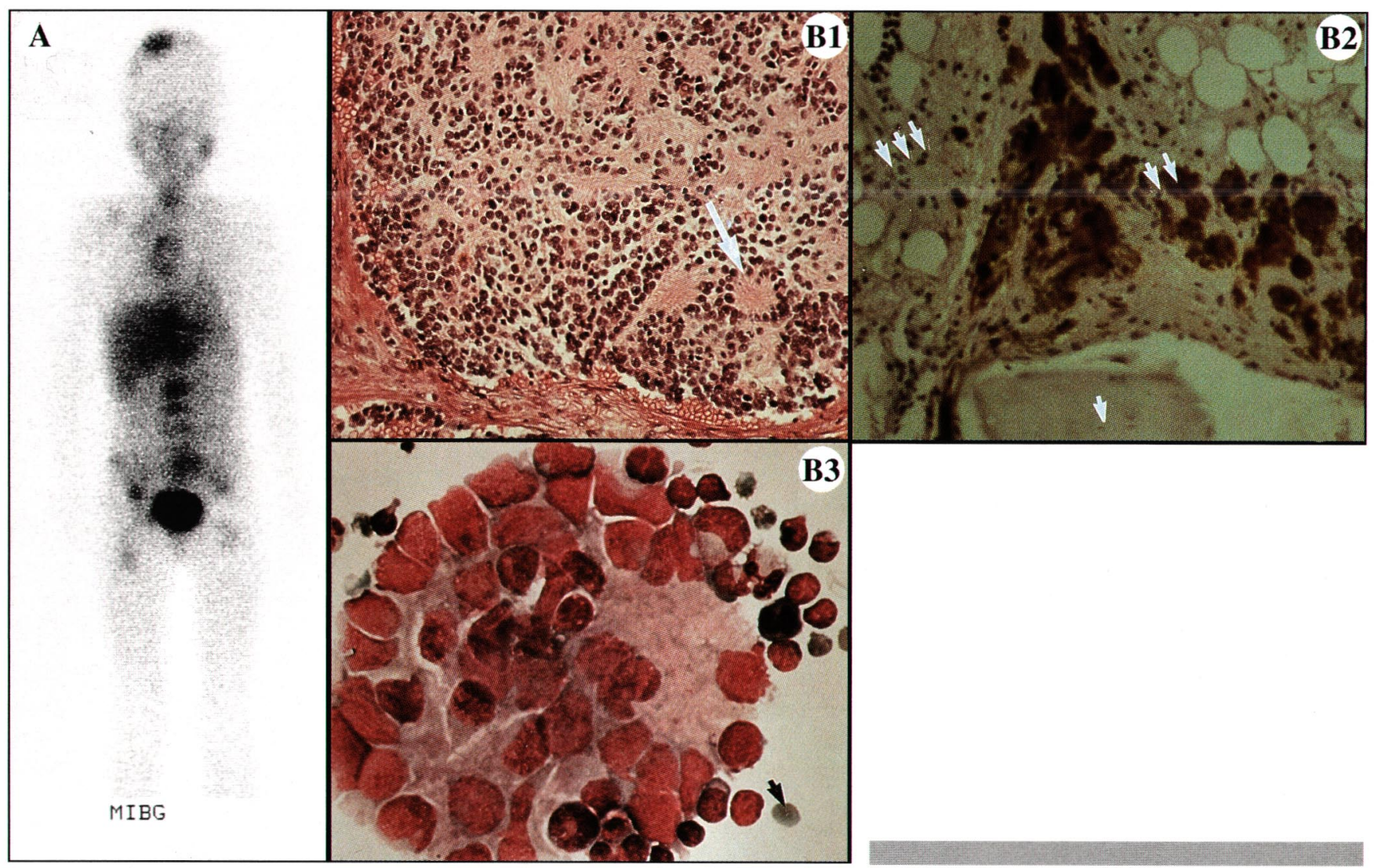

Figure 1. Dissémination métastatique du neuroblastome. A. Scintigraphie osseuse à la mIBG d'un neuroblastome stade IV. L'hyperfixation du marqueur radioactif (méta-iodo-benzy-guanidine, radiomarquée) se fait au niveau de la tumeur primitive (surrénale droite) et des métastases ostéo-médullaires (bassin, rachis et crâne). La faible fixation hépatique, cardiaque et thyroidienne correspond au métabolisme normal du traceur. (Jean Lumbroso, Département Imagerie, IGR, Villejuif). B. Aspects histologiques d'un neuroblastome métastatique. (B1) Tumeur primitive constituée de neuroblastes s'agençant en rosettes (flèche) (coloration hématoxyline-éosine-safran, G X 150). (B2) Métastases osseuses: étude immunohistochimique indiquant une production importante d'énolase (NSE : neuron specific enolase) par les cellules tumorales infiltrant le tissu hématopoïétique (1 flèche, lamelle osseuse; 2 flèches, envahissement tumoral NSE+ ; 3 flèches, tissu hématopoïétique restant). (G x 150). (B3) Métastases médullaires de neuroblastome: groupement caractéristique de cellules neuroblastiques, hématie (1 flèche) (coloration May Grunwald Giemsa, $G \times 700)$.

d'hétérozygotie dans la région 1 p36, une amplification de $\mathrm{N}-M Y C$ et une expression très faible de TRKA. Chaque type, respectivement associé à un pronostic bon, intermédiaire ou très mauvais, définit une entité nosologique distincte de la maladie. Il semble qu'il n'y ait pas de relation de filiation entre ces trois types, le passage d'un type de neuroblastome à un autre n'ayant encore jamais été mis en évidence.

Depuis 1989, des équipes de l'Institut Gustave Roussy (Villejuif), de l'Institut Curie (Paris) et du Centre Léon Bérard (Lyon), ont démontré génétiques pour la conduite du trai- chirurgical suivi d'une chimiothératement du neuroblastome localisé. pie intensive. Le «mariage» de la bioSous l'égide de la Société Francaise logie moléculaire à la clinique oncod'Oncologie Pédiatrique, une étude por- logique a pu trouver là l'une de ses tant sur plus de trois cents cas docu- premières applications en routine ! mentés de neuroblastomes localisés Si l'amplification de N-MYC et la vient de confirmer la nécessité de diploïdie sont des facteurs de mauprendre en compte, dès le diagnos- vais pronostic pour les neuroblastic, l'amplification de N-MYC et le tomes métastatiques de stade IV-S contenu en ADN des cellules tumo- [10], ils ne sont pas assez discrimirales [9]. Ainsi, un neuroblastome nants pour les neuroblastomes de localisé présentant une hyperdiploï- l'enfant âgé de plus d'un an. Pour les die sans amplification de N-MYC sera tumeurs diploïdes, en effet, le protraité uniquement par chirurgie nostic est identique en présence ou alors qu'un neuroblastome localisé en absence d'amplification de Ndiploïde et avec une amplification de $M Y C$. Pour définir des entités nosolo$\mathrm{N}-M Y C$ bénéficiera d'un traitement giques distinctes de ces formes agres- 


\begin{tabular}{|llll|}
\hline \multicolumn{4}{|c|}{ Tableau I } \\
TYPAGE DU NEUROBLASTOME EN FONCTION DU PROFIL \\
GÉNÉTIQUE ET CLINIQUE \\
\hline
\end{tabular}

\section{RÉFÉRENCES}

11. Boyer B, Jouanneau J, Tucker G, Vallès A, Sastre X, Moens G, Thiery JP. La métastase cancéreuse. médecine/sciences $1990 ; 6$ : 433-42.

12. Hata JI, Ueyama Y, Tamaoki N, Furukawa T, Morita K. Human neuroblastoma serially transplanted in nude mice and metastases. Cancer 1978; 42: 468-73.

13. Ferrandis E, Da Silva J, Riou G, Bénard $\mathrm{J}$. Co-activation of the MDR1 and $M Y C N$ genes in human neuroblastoma cells during the metastatic process in the nude mouse. Cancer Res 1994; 54 : 2256-61.

14. Bettan-Renaud L, Bayle C, Teyssier JR, Bénard J. Stability of phenotypic and genotypic traits during the establishment of a human neuroblastoma cell line, IGR-N-835. Int J Cancer 1989; 44: 460-6.

15. Klein, G, Klein E. Evolution of tumours and the impact of molecular oncology. Nature 1985 ; 315 : 190-5.

16. Holzman D. New genes crowd the horizon, create possibilities. J Natl Cancer Inst 1995; 87: 1108-10.

17. Brison O. Gene amplification and tumor progression. Biochim Biophys Acta $1993 ; 1155: 25-41$.

18. Caron P, Peter M, Van Sluis P, de Kraker J, Laureys G, Michon J, Brugières L, Voûte P, Westerveld A, Slater R, Delattre O, Versteeg R. Evidence for 2 tumour suppressor gene loci on chromosome 1p35-36 involved in neuroblastoma: one probably imprinted, another associated with $\mathrm{N}-m y c$ amplification. Hum Mol Genet 1995; 4 : 535-9.

19. Schweigerer L, Breit S, Wenzel A, Tsunamoto K, Ludwig R, Schwab M. Augmented MYCN expression advances the malignant phenotype of human neuroblastoma cells: evidence for induction of autocrine growth activity. Cancer Res 1990; 50 : 4411-6.

20. Schweigerer L, Fotsis T. Human neuroblastoma cells with enhanced N-myc expression: growth factors and growth inhibitors. In: Schwab M, Tonini GP, Bénard J eds. Human Neuroblastoma: recent advances in clinical and genetic analysis. Harwood Academic Publishers, 1993: 135-43.

21. Wenzel A, Cziepluch C, Hamann U, Schürmann J, Schwab M. The N-myc oncoprotein is associated in vivo with the phosphoprotein Max(p20/22) in human neuroblastoma cells. EMBO J 1991 ; 10 : 3703-12.

22. Ayer D, Kretzner L, Eisenmann R. Mad: a heterodimer partner for Max that antagonizes Myc transcriptional activity. Cell 1993; 72: $211-22$

$m / s n^{\circ} 6-7$, vol. 12 , juin-juillet 96
Les quelques cas de dissémination métastatique observés à partir de xénogreffe de neuroblastome humain chez la souris athymique [12] n'ont pas permis d'établir de modèle d'étude des neuroblastes malins métastatiques. C'est à partir des métastases médullaires d'un malade et de la tumeur primitive d'un autre

L'invasion tumorale et la dissémination métastatique des cancers par voie hématogène mettent en jeu des processus complexes à plusieurs étapes liées entre elles qui conduisent à l'angiogenèse, l'invasion des cellules tumorales, leur entrée dans les vaisseaux, leur transport par le sang, leur arrêt au niveau de l'endothélium vasculaire, leur sortie des vaisseaux et leur prolifération sur le site métastatique [11].

On connaît mal le phénomène de dissémination ostéo-médullaire du neuroblastome de stade IV (figure 1); la présence systématique de métastases lors du diagnostic, indépendante du volume de la tumeur primitive, en fait une maladie diffuse d'emblée. Le processus de dissémination du neuroblastome métastatique est différent de celui de nombreux adénocarcinomes métastatiques de l'adulte, du sein ou du côlon par exemple, dont la dissémination succède à la formation d'une tumeur primitive importante. Il existe donc deux maladies métastatiques distinctes, l'une qui caractérise la plupart des tumeurs solides et qui résulte d'une progression tumorale séquentielle d'un point de vue chronologique, et l'autre que l'on désigne par maladie diffuse d'emblée.

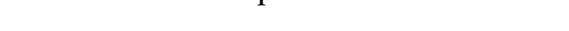

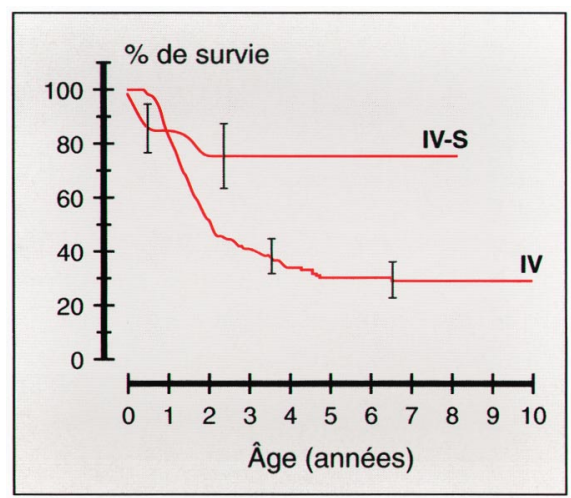

Figure 2. Courbe de survie actuarielle des neuroblastomes métastatiques. Courbe "IV": survie sans événement des neuroblastomes métastatiques d'enfant de plus d'un an ayant bénéficié d'une chimiothérapie intensive suivie d'une autogreffe de moelle osseuse à l'Institut Gustave Roussy ( $n=224)$. (Ellen Benhamou, Département Statistiques Médicales, IGR, Villejuif). Courbe "IV-S ": Survie sans événement de neuroblastomes IV-S $(n=34)$ traités à I'Institut Gustave Roussy [48]. 


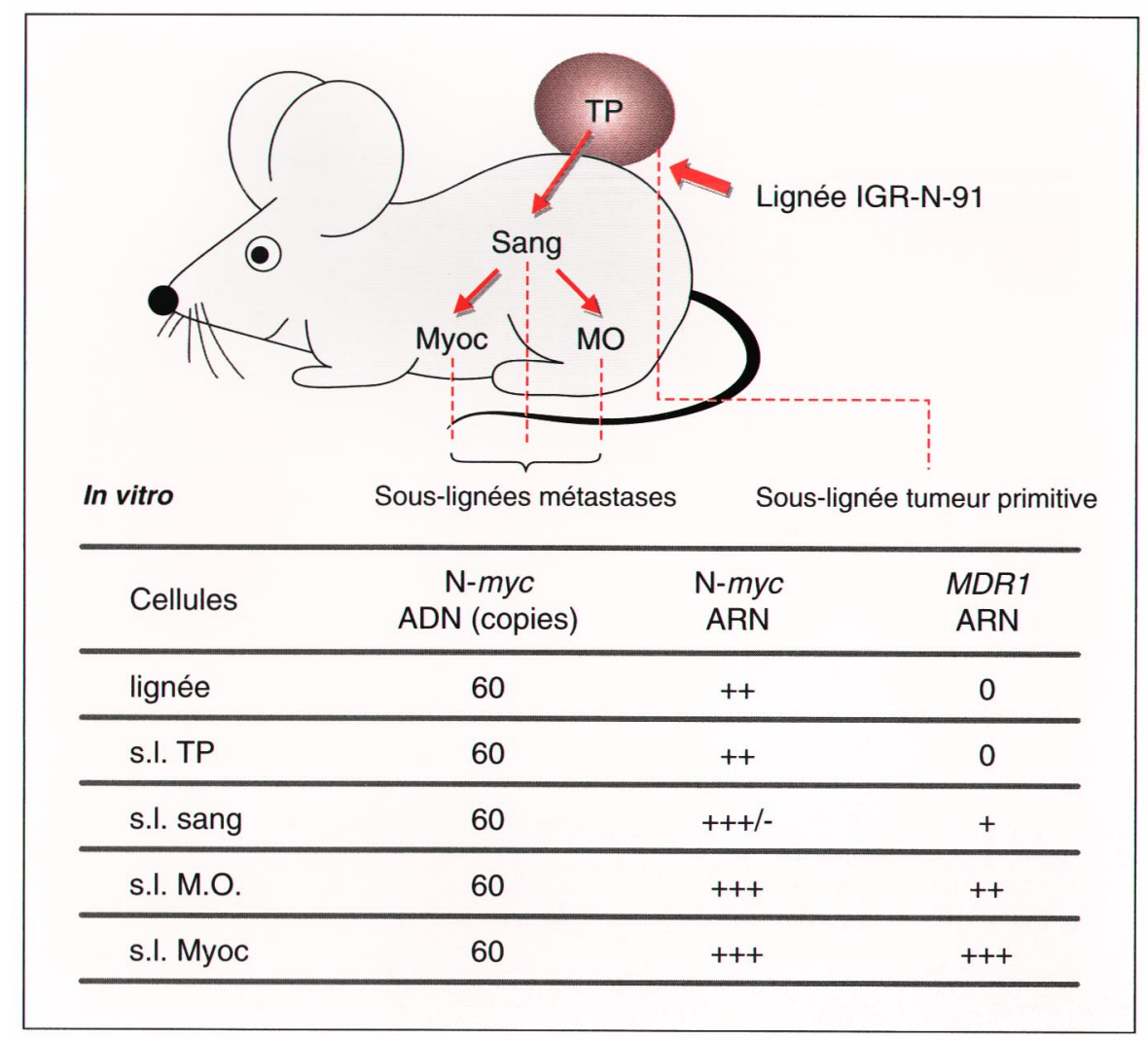

Figure 3. Modèle de neuroblastomes humains disséminant chez la souris athymique. La lignée (I) de neuroblastes malins IGR-N-91 établie in vitro, induit une tumeur "primitive" (TP) sous-cutanée à partir de laquelle des neuroblastes disséminent dans le myocarde (Myoc) et la moelle osseuse (MO) de la souris athymique. La tumeur primitive et les organes sont cultivés in vitro et les différentes sous-lignées (s.l.) sont désignées, respectivement, s.I.TP, s.I. Sang, s.I.MyOc, s.I.MO.

malade que deux lignées de neuroblastomes de stade IV ont été établies, respectivement appelées IGRN-91 [13] et IGR-N-835 [14]. Après injection sous-cutanée à la souris athymique, les cellules IGR-N-91 forment localement des tumeurs hémorragiques très invasives et disséminantes: des métastases macroscopiques sont retrouvées dans les reins, les surrénales des animaux. En outre, des neuroblastes malins sont identifiés dans le sang, la moelle osseuse et le myocarde des souris, neuroblastes qui ont permis d'établir in vitro des sous-lignées [13]. La dissection et l'analyse de la tumeur primitive ont montré que le tissu hémorragique tumoral est à l'origine de la dissémination métastatique (Cappellen et Bénard, communication personnelle). Ces résultats ont été reproduits avec la lignée IGR-N- gènes, des gènes suppresseurs de tumeurs mais aussi des gènes modulateurs [15] impliqués dans le contrôle de l'environnement des clones cellulaires tumoraux, dans les mécanismes de l'angiogenèse et de la dissémination métastatique, et dans le maintien de l'intégrité du génome [16].

\section{Délétion du chromosome 1p, amplification de I'oncogène N-MYC et dissémination métastatique}

A l'exception du type IV-S, le profil génétique des formes métastatiques du neuroblastome, est comparable au type III défini par Brodeur [8]; la perte d'hétérozygotie sur le bras court du chromosome 1 (1p) concerne la moitié des cas [3], et l'amplification de N-MYC, seulement un tiers des cas. La délétion du $1 p$ intéresse toutes les tumeurs avec amplification de N-MYC, et l'association de l'activation d'un oncogène par amplification [17] et de l'inactivation d'un (des) gène (s) suppresseur(s) de tumeur est frappante [7]. Dans $15 \%$ des cas environ, une délétion du $1 p$ est observée en l'absence d'amplification de N-MYC [7]. L'analyse de la perte d'hétérozygotie a permis de réaliser une cartographie fine $\mathrm{du} 1 \mathrm{p}$ entre les régions 1p35 et 1p36-3 et de définir, pour chaque type de tumeur, la zone délétée la plus faible en taille. Il s'avère que, pour les tumeurs avec amplification de $\mathrm{N}-M Y C$, la zone délétée est très large $(1 \mathrm{p} 35 ; 1 \mathrm{p} 36-3)$ et porte, de façon aléatoire, sur le matériel d'origine paternelle ou maternelle. Pour les tumeurs sans amplification de $\mathrm{N}$ $M Y C$, la zone délétée est plus petite et très distale (1p36-2-36-3), et intéresse exclusivement le matériel d'origine maternelle [18]. Ces résultats suggèrent l'existence de deux gènes suppresseurs en 1p35-36.3, dont l'un seulement est soumis à l'empreinte génomique parentale $[7,18]$.

L'existence d'une délétion du $1 p$ dans la tumeur primitive présentant ou non une amplification de N-MYC, suggère que ces altérations ont été acquises avant la dissémination métastatique et confèrent aux neuroblastes leur capacité d'envahir et de 
disséminer. Toutefois, leur existence dans la tumeur primitive ne permet pas de les considérer comme des facteurs discriminants du potentiel métastatique.

\section{RÉFÉRENCES}

23. Bernards R. N-myc disrupts protein kinase C-mediated signal transduction in neuroblastoma. EMBO J 1991; 10 : 1119-25.

24. Bogenmann E, Torres M, Matsushima H. Constitutive N-myc expression inhibits trkA mediated neuronal differentiation. Oncogene 1995; 10 : 1915-25.

25. Galmiche A, Lustenberger P. CD44, invasion tumorale et métastase. médecine/sciences $1994 ; 10: 1282-91$.

26. Shtilvelman E, Bishop JM. Expression of CD44 is repressed in neuroblastoma cells. Mol Cell Biol 1991 ; 11 : 5446-53.

27. Favrot M, Combaret V, Lasset C. CD44-A new prognostic marker for neuroblastoma. N Engl J Med 1993; 329: 1965.

28. Lacombe M, Lascu I, Sastre-Garau X, Wallet V, Véron M. NDP kinases, développement et cancer: une action via des protéines liant le GTP? médecine/sciences 1992; $8: 449-54$.

29. Hailat N, Keim D, Melhem R, Zhu X, Ackerskon C, Brodeur G, Reynolds C, Seeger R, Lottspeich F, Strahler J, Hanash S. High levels of p19/nm23 protein in neuroblastoma are associated with advanced stage disease and with N-myc amplification. I Clin Invest $1991 ; 88$ : 341-5.

30. Leone A, Seeger R, Hong C, Hu Y, Arboleda M, Brodeur G, Stram D, Slamon D, Steeg P. Evidence for $n m 23$ RNA overexpression, DNA amplification and mutation in aggressive childhood neuroblastomas. Oncogene 1993; 8: 855-65.

31. Chang C, Zhu X, Thoravai D, Ungar D, Rawvas J, Hora N, Strahler J, Hanash S, Radany E. nm23-H1 mutation in neuroblastoma. Nature 1994; 370: 335-6.

32. Lepage P, Gros P. La glycoprotéine P: de la résistance croisée aux médicaments au transport des lipides biliaires. médecine/ sciences $1995 ; 11: 357-66$.

33. Bourhis J, Bénard J, Hartmann O, Boccon-Gibod L, Lemerle J, Riou G. Correlation of MDR1 gene expression with chemotherapy in neuroblastomas. J Natl Cancer Inst 1989; 81 : 1401-5.

34. Bénard J, Bourhis J, de Vathaire F, Ferrandis $\mathrm{E}$, Terrier-Lacombe MJ, Lemerle $\mathrm{J}$, Riou G, Hartmann O. Prognostic value of MDR1 gene expression in neuroblastoma: results of a multivariate analysis. In : Evans A, Biedler J, Brodeur G, D'Angio G, Nakagawara A, eds. Advances in neuroblastoma research. New York: Willey-Liss, 1994: 111-6.

35. Ferrandis E, Babajko S, Bénard J. MYCN protein activates the MDR1 promoter in human neuroblasts. Proc Am Ass Cancer Res 1995; 36 : (338 abstr $\left.\mathrm{n}^{\circ} 2010\right)$.

$m / s n^{\circ} 6-7$, vol. 12 , juin-juillet 96 tion chez la souris athymique [13]; en effet, les neuroblastes métastatiques (provenant de la moelle et du myocarde) ont un taux de transcrit de l'oncogène environ double de celui de la tumeur primitive. Notre hypothèse est que l'augmentation de l'expression du gène $\mathrm{N}-M Y C$ entraîne l'activation des gènes impliqués au plan biologique dans ce qu'il est convenu d'appeler "le super décathlon malin » pour décrire ainsi la voie complexe et à étapes multiples utilisée par les cellules métastatiques (invasion, adhérence, migration, etc.). En résumé, la concentration de l'oncoprotéine, qu'elle résulte d'une transfection ou d'une expression constitutive dans des neuroblastes malins, est corrélée à l'angiogenèse et au potentiel métastatique.

Localisée dans le noyau, phosphorylée par la caséine kinase II, la protéine N-Myc, dont la demi-vie est courte, présente dans sa partie carboxy-terminale des motifs structuraux communs à d'autres oncoprotéines-facteurs de transcription comme c-Jun et c-Fos: une région basique de fixation à l'ADN, un domaine hélice-boucle-hélice permettant une interaction protéineprotéine, et un motif à glissière de leucines (leucine zipper). In vivo, dans les neuroblastes malins humains, NMyc, incapable de s'homodimériser, s'associe à la phosphoprotéine Max dont la demi-vie est longue [21]: l'hétérodimère formé N-Myc-Max, capable de se lier à l'ADN sur la séquence consensus CACGTG, transactive certains gènes encore inconnus. Max, à l'inverse, est capable de s'homodimériser et le dimère MaxMax, en se fixant sur la région consensus, réprime l'activité transcriptionnelle de N-Myc. Dans ce contexte, Max étant en excès par rapport à N-Myc (M. Schwab, communication personnelle), c'est la concentration de N-Myc qui conditionnerait l'activité transcriptionnelle de l'oncoprotéine. Il existe, cependant, une autre protéine partenaire, désignée Mad, qui ne forme ni homodimères ni hétérodimères Mad-Myc; en revanche, formant des hétérodimères Mad-Max, elle réprime alors l'activité transcriptionnelle de N-Myc [22]. L'activité de facteur de transcription de N-Myc dépend ainsi entièrement des autres parte- 


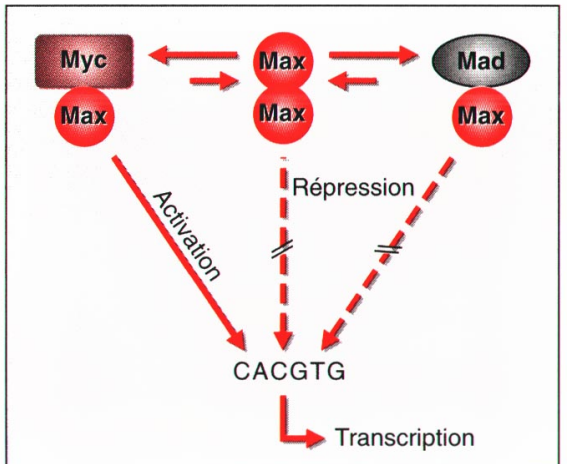

Figure 4. Régulation de I'activité transcriptionnelle de N-MYC dans le neuroblastome. Implication des protéines partenaires. On pense que I'homodimère Max-Max et I'hétérodimère Mad-Max agissent comme des répresseurs alors que l'hétérodimère N-Myc-Max est un activateur transcriptionnel de gènes cibles contenant la séquence consensus CACGTG dans leurs promoteurs. Dans les neuroblastes dans lesquels N-MYC est surexprimé, la formation $d^{\prime}$ 'hétérodimères $N$-Myc-Max est favorisée. L'existence d'homodimères Max-Max et de complexes Mad-Max $n$ 'a pas encore été mise en évidence dans les neuroblastes malins. D'après [22].

naires protéiques (figure 4). Les modèles expérimentaux décrits cidessus représentent actuellement des modèles de choix pour analyser l'influence des variations de concentration de N-Myc, Max et Mad sur les processus d'invasion et de dissémination métastatiques. Ainsi, l'analyse de la protéine $\mathrm{N}-\mathrm{Myc}$ dans des lignées de neuroblastome a permis d'attribuer à cette oncoprotéine non seulement un rôle d'activateur transcriptionnel de gènes impliqués dans la prolifération et la dissémination métastatique mais aussi un rôle de perturbateur de la voie de transduction. Par exemple, les neuroblastes transfectés par N-MYC n'induisent plus l'ARN messager de c-fos ni la synthèse $d u$ facteur transcriptionnel NFkB sous l'action de l'ester de phorbol, activateur de la protéine kinase $\mathrm{C}$; l'induction est restaurée lorsque la protéine kinase $\mathrm{C}$ est introduite dans les neuroblastes transfectés par $\mathrm{N}-M Y C$ [23]. Les auteurs ont montré dans une lignée de neuroblastome murin au potentiel métastatique, B104, provoque la suppression de l'isoforme $\delta$ de la protéine kinase $\mathrm{C}$ et l'induction de son isoforme $\zeta$ [23], inefficace dans cette voie de transduction. D'autre part, dans cette lignée et les sous-lignées transfectées par l'oncogène, l'expression constitutive de N-MYC inhibe la différenciation neuronale relayée par le récepteur du NGF, produit du gène TRKA [24]. En conclusion, il apparaît qu'une expression constitutive de N-MYC inhibe la sortie du cycle cellulaire et bloque la différenciation neuronale [24].

\section{Expression des gènes CD44, NM23 et neuroblastome métastatique}

L'intégrine humaine CD44, glycoprotéine membranaire, est impliquée en tant que récepteur de l'acide hyaluronique, dans la domiciliation lymphocytaire $\mathrm{T}$ et $\mathrm{B}$ et dans l'adhérence à la matrice extracellulaire. Le gène unique exprime une protéine standard $(\mathrm{CD} 44 \mathrm{H})$ et des formes variantes résultant d'un épissage alternatif et d'une modification posttraductionnelle, créant ainsi une famille hétérogène de protéines. Récemment, les isoformes CD44 ont été impliquées dans la formation de métastases (pour une revue générale voir [25]). La forme standard de CD44 accroit les propriétés tumorigènes de mélanomes alors qu'une forme variante particulière, CD44v6, est synthétisée lors de la dissémination métastatique de nombreuses tumeurs humaines solides. La surproduction de CD44v6 est ainsi considérée comme un facteur de mauvais pronostic pour les cancers du côlon, de l'estomac et du sein [25].

A l'inverse, dans le neuroblastome, la dissémination métastatique n'est pas associée à la synthèse d'isoformes variantes ou de CD44 standard. Un travail initial avait même rapporté que l'expression de $C D 44$ était faible ou nulle dans des lignées établies in vitro [26], allant jusqu'à suggérer que la répression du gène pourrait être un marqueur d'agressivité. Ce résultat a été confirmé en analysant des tumeurs de neuroblastome par immunohistochimie ou hybridation sur réplique d'ARN. L'isoforme standard est synthétisée normalement dans $100 \%$ des stades I, II et III, dans le stade IVS et dans les ganglioneuromes mais exceptionnellement dans les tumeurs de stade IV. Quel que soit le type de neuroblastome, les isoformes variantes ne sont pas produites. Dans le neuroblastome métastatique, en revanche, la répression du gène codant pour CD44 est considérée comme un marqueur d'agressivité. Une étude multivariée a confirmé ces résultats en établissant que l'expression de $C D 44$ était un facteur indépendant de bon pronostic [27]. Il s'agit maintenant de comprendre le rôle joué par l'isoforme standard dans la pathogénie du neuroblastome.

Le gène $N M 23$, suppresseur de métastases, existe sous deux formes, $\mathrm{H} 1$ et $H 2$, codant, respectivement, pour les nucléotides diphosphokinases $\mathrm{A}$ et $\mathrm{B}$, protéines homologues impliquées très probablement dans la prolifération, le développement cellulaire, et l'apoptose [28]. Alors que l'expression du gène $N M 23$ est inversement associée au potentiel métastatique dans un grand nombre de cancers humains (sein, mélanome, cancer colique héréditaire), la situation est plus complexe dans le neuroblastome: les formes avancées (stades III et IV) présentent des quantités élevées de transcrits du gène $N M 23 H 1$ avec une amplification du gène pour environ le tiers d'entre elles, associée à un mauvais pronostic [29]. Des études de conformation de l'ADN simple brin et le séquençage direct des parties 5' et 3' traduites des gènes $H 1$ et $H 2$ ont révélé plusieurs altérations: une substitution $\mathrm{Leu}^{48} \rightarrow$ Val, dans la partie amino-terminale de la protéine NM23 [30]. Un travail récent a mis en évidence, dans la partie codante du gène NM23H1, une substitution $\operatorname{Ser}^{120} \rightarrow$ Gly, substitution intéressant 6 des 28 tumeurs de stades III et IV analysées [31] mais que nous n'avons pas retrouvée dans les tumeurs avancées d'une série de 35 tumeurs primitives de neuroblastomes (A. Catteau et al., communication personnelle). Les quelques mutations du gène NM23 décrites à ce jour, pourraient rendre compte du dysfonctionnement de ce gène suppresseur de métastases dans 
le neuroblastome, indépendant d'une réduction de son expression.

\section{RÉFÉRENCES}

36. Moscow J, Fairchild C, Madden M, Ransom D, Wieand H, O'Brien E, Poplack D, Cossmann J, Wyers C, Cowan K. The expression of anionic glutathione-S-tranferase and P-glycoprotein genes in human tissues and tumors. Cancer Res 1989; 49: 1422-8.

37. Lowe S, Ruley H, Jacks T, Houssman D. p53-dependent apoptosis modulates the cytotoxicity of anticancer agents Cell 1993; 74: 957-67.

38. Solary E, Bertrand R, Pommier Y. Le rôle de l'apoptose dans la genèse des cancers. médecine/sciences 1993; 9: 667-75.

39. Fisher D. Apoptosis in cancer therapy: crossing the threshold. Cell 1994; 78 : $539-42$.

40. Vogan K, Bernstein M, Leclerc J, Brisson L, Brossard J, Brodeur G, Pelletier J, Gros, $P$. Absence of p53 gene mutations in primary neuroblastoma. Cancer Res 1993; 53 : 5269-76.

41. Davidoff A, Pence J, Shorter N, Iglehart $\mathrm{J}$, Marks J. Expression of p53 in human neuroblastoma and neuroepithelioma derived cells lines. Oncogene 1992; 7 : 127-33.

42. Moll U, Riou G, Levine A. Two distinct mechanisms alter p53 in breast cancer: mutation and nuclear exclusion. Proc Natl Acad Sci USA 1992; 89 : 7262-6.

43. Moll U, La Quaglia M, Bénard J, Riou G. Wild-type p53 protein undergoes nuclear exclusion in undifferentiated neuroblastomas but not in differentiated tumors. Proc Natl Acad Sci USA 1995; 92: 4407-11.

44. Goldman S, Chen C, Kastan M. Localization and function of p53 in human neuroblastoma. Proc Am Ass Cancer Res 1995; 36 : 610 (abstr $\left.n^{\circ} 3631\right)$.

45. Hanada M, Krajewski S, Tanaka S, Cazals-Hatem D, Spengler B, Ross R, Biedler J, Reed J. Regulation of bcl-2 oncoprotein levels with differentiation of human neuroblastoma cells. Cancer Res 1993; 53 : 4978-86.

46. Hanania N, Boyano MD, Mangin C, Poupon MF. Oncogene and MDRI gene expression in rat rhaddomyosarcoma sublines of different metastatic potential. Anticancer Res 1991 ; 11 : 473-80.

47. Kerbel R, Kobahyashi H, Graham C. Intrinsic or acquired drug resistance and metastasis: are they linked phenotypes? J Cell Biochem 1994; 56 : 37-47.

48. Suarez A, Hartmann O, Vassal G, Giron A, Habrand JL, Valteau D, Brugières L, Khalifa C, Lemerle J. Treatment of stage IV-S neuroblastoma: a study of 34 cases treated between 1982 and 1987. Med Pediatr Oncol 1991; 19: 473-7.

$m / s n^{\circ} 6-7$, vol. 12 , juin-juillet 96 logiques reconnus de la maladie, la surexpression du gène $M D R 1$ est apparue significativement associée à un risque élevé de mortalité [34], résultats confirmés par l'équipe de Victor Ling à Toronto (Canada). Dans notre série, une sous-population de neuroblastomes métastatiques, très agressifs et réfractaires à la chimiothérapie, présentait aussi une amplification de l'oncogène $\mathrm{N}$ $M Y C$. La question posée était de déterminer si la surexpression du gène $M D R 1$ résultait du traitement lui-même, de la progression tumorale, ou de la dissémination métastatique associée à l'expression de l'oncoprotéine N-MYC. Pour y répondre, nous avons utilisé le modèle IGR-N-91 dans lequel l'expression constitutive de N-MYC des neuroblastes malins métastatiques est supérieure à celle de la tumeur primitive. Dans ce modèle, une augmentation significative des transcrits du gène MDR1 et de la concentration de PgP active est observée lors de la dissémination (figure 3), alors que la lignée dérivant de la tumeur primitive n'exprime pas le gène MDR1 [13]: les neuroblastes circulants ayant métastasé dans la moelle et dans le cour expriment le gène de manière croissante et graduée; l'ARNm code pour une PgP fonctionnelle, comme en attestent les mesures de cytotoxicité de l'adriamycine en présence et en l'absence d'inhibiteur de la PgP. Ainsi, l'oncogène $\mathrm{N}-M Y C$ et le gène spécifique de résistance $M D R 1$ sont activés simultanément durant le processus métastatique, et cela en l'absence de toute chimiothérapie. Cette co-activation génique suggérait que la protéine N-Myc active la transcription du gène $M D R 1$, hypothèse qui a pu être confirmée par des expériences réalisées in vitro [35]. L'oncoprotéine serait ainsi capable d'activer la transcription d'un gène spécifiquement responsable d'un phénotype de résistance à la chimiothérapie.

Le gène MDR1 n'est pas le seul déterminant potentiel de la chimiorésistance du neuroblastome. En réponse à l'effet cytotoxique de médicaments tels que le cisplatine et le cyclophosphamide, utilisés dans le traitement de ce cancer, les neuroblastes malins mobilisent un méca- 
nisme de détoxification cytoplasmique impliquant le glutathion et les glutathion-S-transférases. En accord avec l'activation fréquente de la glutathion-S-transférase (GST $\pi$ ) dans les tumeurs humaines [36], nous avons détecté une augmentation de l'expression de ce gène dans les neuroblastomes et prouvé qu'elle n'était pas un indicateur de mauvaise réponse à la chimiothérapie. En revanche, la surexpression combinée des gènes GST $\pi$ et $M D R 1$ apparaît significativement liée à une mauvaise réponse de la tumeur primitive à la chimiothérapie, relation qui reste à démontrer dans les métastases des patients. Les résultats obtenus avec le modèle IGR-N-91 suggèrent que donc que l'activation concomitante de la transcription de ces gènes soit nécessaire à l'installation du phénotype $M D R$ dans les neuroblastes métastatiques.

\section{Les expressions respectives des gènes N-MYC, BCL-2 et P53 et la réponse des neuroblastomes à la chimiothérapie}

Les études récentes sur l'apoptose et en particulier l'action inductible de certains médicaments anticancéreux ([37], pour une revue générale [38]) ont conduit à réviser nos conceptions relatives aux mécanismes de la cytotoxicité des médicaments. Celleci dépendrait de la formation d'un complexe molécule cible, de la dégradation des cibles (ADN, ARN, protéines) mais aussi de l'activation de l'apoptose. En outre, la nature du cancer apparaît également comme un élément déterminant dans la réponse à la chimiothérapie. Les tumeurs testiculaires et certains cancers pédiatriques (leucémie, lymphomes, tumeurs de Wilms) fondent et guérissent de façon spectaculaire, à l'inverse des cancers pulmonaires, colorectaux et cérébraux, à très faible curabilité [39]. Cette grande hétérogénéité de réponse des cancers à la chimiothérapie pourrait refléter une aptitude différente de chaque type tumoral à engager les cellules vers l'apoptose.

Les données récentes obtenues sur des modèles cellulaires - essentielle-
P53 [37, 39], c-MYC et BCL-2 [38] comme les acteurs-clés du contrôle génétique de ces processus : l'expression respective de ces gènes orienterait la cellule vers la pause réparatrice, la prolifération ou l'apoptose [38]. Dans le cas du neuroblastome, le gène $P 53$ est actuellement le mieux connu. Si les mutations du gène $P 53$ sont très rares dans les tumeurs de neuroblastomes [40], on observe une surexpression du gène dans des neuroblastes immatures établis en culture [41]. Ainsi beaucoup d'auteurs ont conclu à la non- implication du gène $P 53$ dans l'étiologie et la progression du neuroblastome. On peut néanmoins émettre l'hypothèse que, dans ce cancer, la p53 serait inactivée par un autre mécanisme, impliquant la participation de protéines capables de la séquestrer, au niveau nucléaire comme la protéine nucléaire $\mathrm{Mdm} 2$, ou au niveau cytoplasmique comme cela a été démontré dans le cancer du sein [42]. En collaboration avec Ute Moll de Stony Brook à New York, nous venons de montrer qu'en l'absence d'amplification du gène $M D M 2$, la plupart des tumeurs indifférenciées de neuroblastome présentent une augmentation de la concentration de la protéine p53 dans le cytoplasme [43], ces données suggérant un défaut dans le transport de la p53 du cytoplasme vers le noyau. Qu'il s'agisse d'un mécanisme de séquestration (par quelle protéine?), ou d'une modification de la p53, l'exclusion nucléaire conduirait à la perte de fonction de la protéine. Il vient d'être rapporté que l'irradiation de neuroblastes cultivés in vitro et présentant une p53 cytoplasmique, fait apparaître, dans le noyau, une p53 fonctionnelle [44]. Ces données pourraient conduire au développement d'une thérapeutique combinée et séquentielle chimiothérapie-radiothérapie du neuroblastome métastatique.

En ce qui concerne l'implication de l'oncoprotéine Bcl-2 dans la pathogénie du neuroblastome, des travaux réalisés sur des lignées indiquent que le gène de la protéine cytoprotectrice est surexprimé dans les neuroblastes immatures et non exprimé dans les cellules différenciées [45]. Paradoxalement, la participation de l'oncoprotéine N-Myc à l'apoptose reste mal connue. Aussi, afin d'éva- luer l'implication de ces gènes dans le processus apoptotique et la réponse des neuroblastes malins à une chimiothérapie, le statut des gènes $P 53$, BCL2 et N-MYC dans les modèles expérimentaux «tumeur-métastases» est en cours d'étude dans notre laboratoire.

\section{Perspectives}

Nos connaissances sur les altérations génétiques impliquées dans les processus métastatiques du neuroblastome sont très limitées. Sans données physiopathologiques sur la dissémination métastatique, il est difficile de déterminer si des anomalies génomiques importantes portant sur les oncogènes et les gènes suppresseurs de tumeurs, représentent des événements antérieurs ou postérieurs à la dissémination. En revanche des altérations de structure et d'expression des gènes «modulateurs" tels $C D 44$ et NM23 semblent être plus spécifiquement associées au processus métastatique. On peut espérer que les modèles expérimentaux de neuroblastome humain permettront de préciser ces données.

Chez les patients dont la tumeur présente une amplification de $\mathrm{N}$ $M Y C$, comme dans le modèle expérimental de neuroblaste humain IGR-N-91, l'accroissement de l'expression de l'oncogène $\mathrm{N}-M Y C$ est un facteur de stimulation de l'angiogenèse et de l'invasion, de la dissémination métastatique et de l'expression d'un phénotype de chimiorésistance. Si cette activation oncogénique n'intéresse qu'un nombre limité des neuroblastomes métastatiques ( $1 / 4$ environ), elle doit être néanmoins rapprochée d'observations antérieures relatives au rhabdomyosarcome métastatique du rat [46] où c-JUN, c-FOS et c- $M Y C$, gènes codant pour des protéines oncogéniques qui jouent aussi un rôle de facteur transcriptionnel, sont activés en parallèle avec le gène MDR1 et contrôlent l'expression du potentiel métastatique de ces modèles tumoraux. Ainsi, apparaît un lien direct entre la dissémination métastatique et la résistance aux médicaments anticancéreux [47].

A la lumière des données présentées dans cette revue, on peut suggérer que les niveaux de synthèse d'onco- 
protéine(s) et protéine(s) suppressive(s) contrôlant le cycle cellulaire et l'apoptose déterminent les processus de dissémination métastatique et de chimiorésistance des neuroblastomes. Une connaissance plus fine de la régulation de ces protéines nucléaires pourrait conduire à une classification génétiques des neuroblastomes métastastatiques et permettre des approches thérapeutiques différentes de celles de la chimiothérapie conventionnelle

TIRÉS À PART

J. Bénard.

\section{Summary}

Genes involved in metastatic dissemination and response to chemotherapy in neuroblastoma

Several genetic defects found in neuroblastoma are associated with the poor prognosis of this childhood cancer: deletion of the short arm of chromosome 1, N-MYC amplification and the absence of CD44 gene expression. However, evidencing these anomalies in initially metastatic primary neuroblastoma did not allow for improving the treatment of this aggressive form of the disease with a low survival. Alterations specifically associated with the potential for metastatic dissemination have thus been sought for, in an attempt to classify metastatic neuroblastoma. In an experimental model of metastatic human neuroblastoma in athymic mice, $\mathrm{N}-M Y C$ overexpression was demonstrated to be a marker of metastatic dissemination. Moreover, an increase in the rate of the oncoprotein in metastatic neuroblasts was found to be associated with genes responsible for the MDR phenotype (MDR1, GST- $\pi)$. The combined activity of N-Myc, Bcl-2 and wild type p53 sequestered in the cytoplasm seems to promote an antiapoptotic mechanism. These anomalies could explain the often observed resistance to intensive chemotherapy and should lead to the promotion of new treatments for metastatic neuroblastoma. 